\title{
Secure Outsourcing of DNA Searching via Finite Automata
}

\author{
Marina Blanton and Mehrdad Aliasgari \\ Department of Computer Science and Engineering, University of Notre Dame, \\ \{mblanton,maliasga\}@cse.nd.edu
}

\begin{abstract}
This work treats the problem of error-resilient DNA searching via oblivious evaluation of finite automata, where a client has a DNA sequence, and a service provider has a pattern that corresponds to a genetic test. Error-resilient searching is achieved by representing the pattern as a finite automaton and evaluating it on the DNA sequence, where privacy of both the pattern and the DNA sequence must be preserved. Interactive solutions to this problem already exist, but can be a burden on the participants. Thus, we propose techniques for secure outsourcing of finite automata evaluation to computational servers, which do not learn any information. Our techniques are applicable to any type of finite automata, but the optimizations are tailored to DNA searching.
\end{abstract}

\section{Introduction}

The need to protect private or sensitive information about an individual is widely recognized. Recent advances in bioinformatics and biomedical science promise great potential in our ability to understand and compute over genome data, but the DNA of an individual is highly sensitive data. In recent years, several publications appeared that allow for computing over DNA data in a private manner with the purpose of identifying ancestry relationships or genetic predisposition. In particular, results are known for sequence comparisons that compute the edit-distance 12, error-resilient pattern matching based on finite automata (FA) evaluation [34, and specific DNA-based ancestry testing [5].

DNAs or DNA fragments used in such computations are large in size. For that reason, recent work [26] concentrated on improving the efficiency of such protocols, but they still remain resource-intensive. Thus, if a customer would like to engage in a private computation that uses her DNA, she might not have computational resources or bandwidth to carry out the protocol. When this is the case, it is natural to consider outsourcing the computation to powerful servers or a large distributed network such as a computational grid. Obviously, in such a setting the privacy of all sensitive inputs (the customer's DNA, the service provider's tests, etc.) must be preserved from the participating servers.

Results for privacy-preserving outsourcing of the edit distance computation of two strings are known [7/8], but outsourcing of more general type of computation over DNA via finite automata has remained unexplored. Thus, the focus of this work is on secure outsourcing of oblivious evaluation of a finite automaton on 
a private input. We use the work of Troncoso-Pastoriza et al. that pioneered techniques for oblivious finite automata evaluation (OFAE) [3] as a starting point for out solution and develop techniques for outsourcing such computations.

Using FA for DNA searching is motivated by the fact that queries on DNA data need to take into account various errors such as clinically irrelevant mutations, sequencing errors, incomplete specifications, etc. Such errors can be tolerated if the pattern is expressed using regular expressions, implemented as FA. We refer the reader to 3] for a detailed description of searching and alignment algorithms that can be implemented using FA. Then a service provider (such as, e.g., 23andMe 9]) can build a FA that implements a genomic test, and a customer who possess a private DNA sequence will use it as an input to the automaton. A DNA sequence is specified as a string of characters over the alphabet $\Sigma=\{A, C, T, G\}$ of length $N$, and a deterministic finite automaton (or a finite state machine (FSM)) corresponding to a DNA test is specified as a tuple $M=\left(Q, \Sigma, \Delta, q_{0}, F\right)$, where $Q$ is a set of states, $\Sigma$ is an alphabet, $\Delta: Q \times \Sigma \rightarrow Q$ is the transition function, $q_{0} \in Q$ is the initial state, and $F \subseteq Q$ is the set of final states. W.l.o.g, the transition matrix is assumed to be complete, i.e., it specifies a transition from each state on each input, and is represented as a table of size $|Q| \times|\Sigma|$, where each value stores a state. The states are represented as integers in $\mathbb{Z}_{|Q|}$ and input characters are represented as integers in $\mathbb{Z}_{|\Sigma|}$. A FA $M$ accepts a string $x=x_{0} x_{1} \ldots x_{N-1} \in \Sigma^{N}$ if on input $x$ it transitions from $q_{0}$ to $q_{N} \in F$.

Contributions. Our contributions can be summarized as follows:

- We first show how the solution of 3 can be simplified to improve both the computation and communication for typical values of the parameters (i.e., when $|\Sigma|$ is small). We also provide a detailed (not just asymptotic) analysis of the original and modified solutions and show that the communication cost can be rather high and not suitable for all clients. Since most of the communication overhead of the solutions comes from the oblivious transfer (OT) protocol, we analyze the performance of the solutions using different OT realizations that allow us to achieve a computation-computation tradeoff.

- We give a protocol for outsourcing the computation of both the client and the FA owner (service provider) to two computational servers without increasing either the communication or computational complexity of the protocol. The communication complexity of the client and service provider becomes linear in the size of their data and involves virtually no computation.

- Next, we give a protocol that works for outsourcing the computation to any number of servers (i.e., the multi-party case). To minimize the overhead, we use a different structure from that used in the two-party outsourcing solution. To lower the communication complexity (and in part the computation overhead), we represent the transition matrix $\Delta$ as a square, so that the communication is decreased from $O(|\Sigma|+|Q|)$ to $O(\sqrt{|\Sigma||Q|})$.

- We also develop a threshold version of the multi-party outsourcing protocol which makes the solution suitable to work in unstable or dynamic environments such as grids. Due to space limitation, it could not be included in this article and can be found in the full version [10]. 


\section{Related Work}

There is a considerable number of publications on secure DNA comparison and matching (e.g., 18113512]). The majority of them (e.g., 17826]) use dynamic programming (DP) to securely compute the edit distance between a pair of genomic sequences: There are two parties, each with its respective sequence, and the algorithms compute the edit distance between the sequences without revealing any information besides the output. Since the DP techniques involve computation quadratic in the input size, such solutions are computation and communication heavy. For that reason, consecutive work [78 considered outsourcing the edit distance computation to powerful helper servers, and another line of research [26] concentrated on making such solutions more efficient. Related to them, 13. gives secure computation of the longest common subsequence (LCS) using optimized techniques, and this research is continued in [14.

While these techniques are likely to improve the communication and/or computation complexity of the original DP solution, one might consider the edit distance computation to be a specific type of DNA comparison that might not be suitable when, e.g., error-resilient searching is necessary (handling sampling errors, incomplete specifications, etc.). For that reason, another line of research 34 3 uses FSMs to implement error-resilient searching over DNA data, and can support any searches that can be formulated as regular languages. These publications provide secure two-party protocols for OFAE, which can be used in any context and is not limited to DNA searching. We use the first publication in this domain [3] as a starting point for our outsourcing construction. A follow-up work [4] uses techniques similar to generic Boolean circuit evaluation to significantly lower the round complexity of the protocol (from $O(N)$ to $O(1)$ ) and lower the computation complexity as well. The circuit-based approach, however, does not generalize to the outsourcing scenario, since it assumes that the function to be evaluated (i.e., a FA in our case) is known to the participants. Other general secure function evaluation approaches are not suitable for the same reason.

Other work on privacy-preserving computing over DNA data includes [1], where the authors introduce a strategy for enhancing data privacy in a distributed network deploying the Smith-Waterman algorithm for sequence comparison. In [5], the authors build secure multi-party protocols for specific genetic tests such as parental tests; the approach can also handle a small number of errors, but the complexity of the protocol rapidly increases with the number of errors it can tolerate. Lastly, 12 presents a cryptographic framework for executing queries on databases of genomic data, where data privacy is achieved by relying on two non-colluding third parties.

\section{Preliminaries}

Homomorphic encryption. Prior and our work relies on a semantically secure homomorphic public-key encryption scheme. Let $\mathcal{E}=$ (Gen, Enc, Dec) be a public-key encryption scheme, where key generation algorithm Gen takes a security parameter $1^{\kappa}$ and produces a public-private key pair $(p k, s k)$; encryption 
algorithm Enc takes $p k$ and message $m$ and produces a ciphertext $c$; and decryption algorithm Dec takes $(p k, s k)$ and ciphertext $c$ and produces $m$. For brevity, we use notation $\operatorname{Enc}_{p k}(m)$ and $\operatorname{Dec}_{s k}(c)$. Let $n$ be the public modulus associated a public key $p k$; the message space is then $\mathbb{Z}_{n}^{*}$. We will assume that $|n|=\kappa$.

With homomorphic encryption, operations on ciphertexts translate into certain operations on the underlying plaintexts. For additively homomorphic schemes, $\operatorname{Enc}_{p k}\left(m_{1}\right) \cdot \operatorname{Enc}_{p k}\left(m_{2}\right)=\operatorname{Enc}_{p k}\left(m_{1}+m_{2}\right)$, which implies $\operatorname{Enc}_{p k}(m)^{a}=\operatorname{Enc}_{p k}(m a)$ for known $a$. A ciphertext $\operatorname{Enc}_{p k}(m)$ can be re-randomized by multiplying it to $\mathrm{Enc}_{p k}(0)$; this makes it infeasible to link the new ciphertext to the original one.

Oblivious transfer. A 1-out-of-t oblivious transfer, $\mathrm{OT}_{1}^{t}$, allows the receiver to retrieve one item from the $t$ items at the sender in a way that the receiver does not learn anything besides that item and the sender learns nothing. It is a well studied cryptographic tool, with many available realizations. Different OT protocols from the literature allow one to achieve tradeoffs between sender and receiver computation and their communication. That is, $\mathrm{OT}_{1}^{t}$ from [15] has very efficient amortized cost (one modulo exponentiation per OT for the sender and the receiver) and linear communication cost $O(t)$. Other protocols (e.g., [16 17]) achieve sub-linear communication, but have larger computation requirements. Depending on the parameters used in OFAE (i.e., the number of states, input length, etc.) and resources available to the participants, one scheme might be preferred over another. We use different OT schemes in analysis in Section 5 .

Oblivious evaluation of finite automata. Here we review the solution of [3], which is used as a starting point in this work. The service provider $\mathcal{S}$ holds $\Delta$ and the client $\mathcal{C}$ holds input $x$. The evaluation processes one input character at a time, and the current state is shared between $\mathcal{C}$ and $\mathcal{S}$ modulo $|Q|$. Throughout this paper, we will assume that the rows of the matrix are numbered 0 through $|Q|-1$, and the columns of the matrix are numbered from 0 to $|\Sigma|-1$. The solution consists of three sub-protocols: (i) a protocol for performing the first state transition, (ii) a protocol for executing a general $k$ th state transition (for $k=1, \ldots, N-1$ ), and (iii) a protocol for announcing the result to the client. Our description of the (main) $k$ th state transition protocol here is slightly different from its original presentation in [3]: it is described for a transposed matrix to improve efficiency of the protocol (as was suggested in [3]). We use $q_{i}$ to denote the current state in the execution after processing $i$ input characters. Notation $a \stackrel{R}{\leftarrow} A$ means that $a$ is chosen uniformly at random from the set $A$. The protocol uses a homomorphic encryption scheme $\mathcal{E}$ for which only $\mathcal{C}$ knows $s k$.

Protocol for 1st state transition. It allows $\mathcal{C}$ and $\mathcal{S}$ to evaluate the FA on the first input symbol, i.e., compute $q_{1}=\Delta\left(q_{0}, x_{0}\right)$, and share it in an additively split form, i.e., $\mathcal{S}$ learns $q_{1}^{(1)}$ and $\mathcal{C}$ learns $q_{1}^{(2)}$ such that $q_{1}^{(1)}+q_{2}^{(2)} \bmod |Q|=q_{1}$.

1. $\mathcal{S}$ picks $r \stackrel{R}{\leftarrow} \mathbb{Z}_{|Q|}$ and blinds each value in row $q_{0}$ by adding $r$ to it $\bmod |Q|$.

2. The parties engage in an $\mathrm{OT}_{1}^{|\Sigma|}$, where the sender $\mathcal{S}$ uses the blinded row $q_{0}$ as its database and receiver $\mathcal{C}$ retrieves the element at position $x_{0}$.

At the end, $\mathcal{S}$ has $q_{1}^{(1)}=-r \bmod |Q|$ and $\mathcal{C}$ has $q_{1}^{(2)}=q_{1}+r \bmod |Q|$. 
Protocol for $k$ th state transition. Prior to the protocol, $\mathcal{C}$ and $\mathcal{S}$ additively share the $k$ th state (i.e., $\mathcal{S}$ has $q_{k}^{(1)}$ and $\mathcal{C}$ has $q_{k}^{(2)}$ such that $q_{k}=q_{k}^{(1)}+q_{k}^{(2)} \bmod$ $|Q|) ; \mathcal{C}$ also holds the next input character $x_{k}$ and $\mathcal{S}$ holds the transition matrix $\Delta$. The output consists of $\mathcal{C}$ and $\mathcal{S}$ additively sharing the $(k+1)$ st state $q_{k+1}$.

1. $\mathcal{S}$ chooses $r \stackrel{R}{\leftarrow} \mathbb{Z}_{|Q|}$ and blinds each element of $\Delta$ by adding $r$ to the element modulo $|Q|$. $\mathcal{S}$ rotates the matrix $q_{k}^{(1)}$ rows up to obtain modified matrix $\Delta_{k}$.

2. $\mathcal{C}$ generates a binary vector of length $|\Sigma|$ consisting of a 1 at position $x_{k}$ and 0 's in other positions. $\mathcal{C}$ encrypts the vector with $p k$ and sends encrypted bits $e=\left(e_{0}, \ldots, e_{|\Sigma|-1}\right)$ to $\mathcal{S}$, where each $e_{i}=\operatorname{Enc}_{p k}\left(b_{i}\right)$ and $b_{i} \in\{0,1\}$.

3. $\mathcal{S}$ performs matrix multiplication of $e$ and $\Delta_{k}$ using the homomorphic properties of the encryption. As a result, $\mathcal{S}$ obtains a new vector $v=\left(v_{0}, \ldots, v_{|Q|}\right)$, that corresponds to an element-wise encryption of the column at position $x_{k}$.

4. Both parties engage in an $\mathrm{OT}_{1}^{|Q|}$, where the sender $\mathcal{S}$ holds vector $v$ and receiver $\mathcal{C}$ retrieves the element at position $q_{k}^{(2)}$.

5. $\mathcal{C}$ decrypts the value and obtains $q_{k+1}^{(2)} ; \mathcal{S}$ sets its share to $q_{k+1}^{(1)}=-r$.

Protocol for announcement of result. In the beginning of the protocol, $\mathcal{C}$ and $\mathcal{S}$ additively share state $q_{N}$ modulo $|Q|$. As a result of this protocol, $\mathcal{C}$ learns whether the evaluation resulted in an accept state or not, i.e., it learns a bit.

1. $\mathcal{S}$ generates a random binary vector $f$ of length $|Q|$ by setting its element at position $j+q_{N}^{(1)}$ to 1 if the state $j \in F$, and to 0 otherwise.

2. Both parties engage in an $\mathrm{OT}_{1}^{|Q|}$, where the sender $\mathcal{S}$ holds vector $f$ and receiver $\mathcal{C}$ retrieves the element at position $q_{N}^{(2)}$.

\section{Security Model}

The requirements that a scheme for secure outsourcing of OFAE must meet are:

Correctness: The protocol should provide the client with correct evaluation of the service provider's finite state machine $M$ on the client's input $x$.

Efficiency: Communication and computation complexity of $\mathcal{C}(\mathcal{S})$ should be linear in the size of its input $x$ (in the size of the automaton $M$ (i.e., the size of $\Delta)$, respectively). Communication and computation complexity (including round complexity) of the servers should be minimized if possible.

Security: The servers should not learn any information throughout the protocol execution. We assume that the servers are trusted to perform their computation correctly, i.e., they are semi-honest or honest-but-curious in that that they will follow the protocol as prescribed, but might attempt to learn additional information from the intermediate values.

We now can formally define security using the standard definition in secure multi-party computation for semi-honest adversaries. Since the helper servers do not contribute any data to the computation, this should be interpreted as no private input to the function they are evaluating. Then for the purposes of the security definition, all data the servers receive before or during the computation (i.e., the transition matrix and client's input) are considered to be a part of the function and therefore must leak no information. We denote "no data" by $\perp$. 
Definition 1. Let parties $P_{0}, \ldots, P_{m-1}$ engage in a protocol $\pi$ that computes function $f(\perp, \ldots, \perp)=\left(o_{0}, \ldots, o_{m-1}\right)$, where $o_{i}$ denotes output of party $P_{i}$. Let $\mathrm{VIEW}_{\pi}\left(P_{i}\right)$ denote the view of participant $P_{i}$ during the execution of protocol $\pi$. It is formed by $P_{i}$ 's input and any internal random coin tosses $r_{i}$, as well as messages $m_{1}, \ldots, m_{t}$ passed between the parties during protocol execution $\mathrm{VIEW}_{\pi}\left(P_{i}\right)=\left(\perp, r_{i}, m_{1}, \ldots, m_{t}\right)$. We say that protocol $\pi$ is secure against sem $i$ honest adversaries if for each party $P_{i}$ there exists a probabilistic polynomial time simulator $S_{i}$ such that $\left\{S_{i}(f(\perp, \ldots, \perp))\right\} \equiv\left\{\operatorname{VIEW}_{\pi}\left(P_{i}\right), \perp\right\}$, where $\equiv$ denotes computational indistinguishability.

Note that this standard model allows the helper servers to collude (i.e., share the information) in the multi-party case. The security guarantees must hold as long as the coalition size does not exceed a specific threshold. The computational servers do not receive any output, but rather communicate the result to $\mathcal{C}$.

\section{Secure FSM Evaluation}

Before proceeding with outsourcing solutions, we give a simplification of the original approach that simultaneously improves its communication and computation overhead. Our simplification involves representing the matrix $\Delta$ as a one-dimensional list (instead of a two-dimensional table), and does not affect the functionality or security of the solution while allowing us to skip encryption and handling of encrypted data. When we represent the matrix as a list, we reference element $(i, j)$ of the matrix as the element at index $|\Sigma| i+j$ in the list.

Protocol for 1st state transition. The same as before.

Protocol for $k$ th state transition. Prior the protocol, $\mathcal{C}$ and $\mathcal{S}$ additively share the $k$ th state modulo $|Q|$, and the output of the protocol consists of $\mathcal{C}$ and $\mathcal{S}$ additively sharing the $(k+1)$ st state.

1. $\mathcal{S}$ chooses $r \stackrel{R}{\leftarrow} \mathbb{Z}_{|Q|}$ and blinds each element of $\Delta$ by adding $r$ to it $\bmod |Q|$.

2. $\mathcal{S}$ rotates the matrix $\Delta q_{k}^{(1)}$ rows up. Let $\Delta_{k}$ denote the modified matrix. $\mathcal{S}$ then represents $\Delta_{k}$ as a list of $|Q| \cdot|\Sigma|$ elements.

3. $\mathcal{C}$ and $\mathcal{S}$ engage in $\mathrm{OT}_{1}^{|Q| \cdot|\Sigma|}$, at the end of which $\mathcal{C}$ obtains the element at position $|\Sigma| \cdot q_{k}^{(2)}+x_{k}$ from the list corresponding to $\Delta_{k}$.

Protocol for announcement of result. The same as before.

We now can compare performance of the protocol above with the original solution from [3]. As suggested in [3], we assume that the efficient $\mathrm{OT}_{1}^{t}$ protocol with amortized single exponentiation per transfer [15] is used. Also, since in this application $|\Sigma| \ll|Q|$, we assume that the transition matrix is transposed (as presented in Section 3) to result in maximal savings from the OT protocol.

In the analysis, we include all modular exponentiations and also count modular multiplications if their number is large; the overall complexity is expressed in the number of modular exponentiations $(1 \bmod \exp =\kappa \bmod \operatorname{mult})$. The results for $k$ executions of the $k$ th state transition protocol are presented in 
Table 1. Analysis of original and modified oblivious automata evaluation solutions

\begin{tabular}{|l|c|c|}
\hline & Original [3] & Modified \\
\hline $\mathcal{C}$ 's exps & $(|\Sigma|+2) N$ & $N$ \\
\hline $\mathcal{S}$ 's exps & $Q \mid+N(1+(\log (|Q|)|\Sigma|+|\Sigma|-1)|Q| / \kappa)$ & $|Q||\Sigma|+N(1+|\Sigma||Q| / \kappa)$ \\
\hline Comm & $2 \kappa N(|\Sigma|+|Q|)$ & $\log (|Q|) N|\Sigma||Q|$ \\
\hline
\end{tabular}

Table 1 (the rest of the work is much lower). In the original scheme, in each protocol round, $\mathcal{C}$ performs $|\Sigma|$ encryptions, 1 decryption, and 1 exponentiation (for the OT). $\mathcal{S}$ 's work for $N$ OT protocols involves $|Q|+1$ exponentiations and $N|Q|$ multiplications. To process the client's response in each round, it performs $|Q \| \Sigma|$ exponentiations with small exponents (or length $\log |Q|$ ), which results in $N(\log (|Q|)|\Sigma|) / \kappa$ regular modular exponentiations overall. Since the client sends $|\Sigma|$ encrypted values and the OT protocol involves the transfer of $|Q|$ encrypted messages in each round, the overall communication is $2 \kappa N(|\Sigma|+|Q|)$.

In the modified scheme, only OT is used, and thus $\mathcal{C}$ 's work drops by a factor of $|\Sigma|+2$. S's work is also lowered, as the dominating term in the original solution is $|Q \| \Sigma| N \log (|Q|) / \kappa$, while in the modified scheme it is $|Q \| \Sigma| N / \kappa$. This means that the server's work drops by a factor of $\log |Q|$ (which is an improvement by at least an order of magnitude). Even though the communication complexity is now proportional to $N|\Sigma||Q|$ instead of $N(|\Sigma|+|Q|)$ in the original protocol, it can be two orders of magnitude lower due to the overhead caused by the security parameter $\kappa$ in the original scheme (i.e., for any feasible finite automaton size, $\log |Q| \ll \kappa$; a typical setup can consist of $\log (|Q|) \leq 20,|\Sigma|=4$, and $\kappa=1024)$.

One of our original motivations for this analysis was large communication overhead of the scheme. For instance, genome sequences can be billions of characters long, but even with the current ability to sample them, the sequences are in the thousands. A FSM that represents a search pattern can have significantly more states than the length of the pattern itself due to the need to handle errors. Thus, for a sample setup of $N=10,000,|Q|=50,000$, and $\kappa=1024$, the communication cost of the original solution is $10^{12}$ bits $\approx 0.125 \mathrm{~TB}$ (it is lowered to $\approx 3 \cdot 10^{10}$ bits in the modified solution). Such overhead is prohibitively large for many clients (e.g., it can take several days or even months on a rather fast DSL link). Thus, we investigate the use of other OT protocols, which can lower the communication overhead of the protocol. Then depending on the computational power and the bandwidth one has, the most suitable choice can be used.

Besides existing OT protocols, the OT functionality can be achieved by utilizing a Private Information Retrieval (PIR) protocol, which differs from OT in that the receiver may learn additional information about the database besides the item or block it receives. Transferring a PIR protocol to a Symmetric PIR (in which privacy of the database is also preserved, and the receiver learns only its item) can be done at low cost using the techniques from [18] or [19], which will give us an OT protocol. We compare the performance of OFAE using three efficient PIR protocols of different nature. In particular, several PIR protocols (e.g., 20 2116 17] ) were studied in [22], and we select most communication 
Table 2. Performance of the original OFAE protocol (except matrix multiplication) using different OT protocols

\begin{tabular}{|c|c|c|c|}
\hline & Lipmaa OT & GR OT & AG OT \\
\hline $\mathcal{C}$ 's op. & $K_{1} N \log (|Q|)(\log (|Q|) / 2-1)$ & $\left(4 N K_{e} \sqrt{|Q|}\right)$ & $N\left(K_{3}^{\log 10}+2 K_{3}^{\log 5}+|Q| K_{e}\right)$ \\
\hline $\mathcal{S}$ 's op. & $(2|Q|-\log (|Q|)) K_{1} N$ & $2|Q| K_{e} N$ & $N K_{3}^{2}$ \\
\hline Comm & $\begin{array}{c}N\left(\left(K_{1} / 2\right) \log ^{2}(|Q|)+\right. \\
\left.+3 K_{e} \log (|Q|)\right)\end{array}$ & $\begin{array}{c}N\left(\log (|Q|)+K_{e}+4\right. \\
+\log (\log (|Q|)))\end{array}$ & $N|Q| K_{e} K_{3}^{2}$ \\
& & \\
\hline
\end{tabular}

Table 3. Performance of the simplified OFAE protocol using different OT protocols

\begin{tabular}{|c|c|c|c|}
\hline & Lipmaa OT & GR OT & AG OT \\
\hline $\mathcal{C}$ 's op. & $\begin{array}{c}K_{1} N \log (|Q||\Sigma|) \times \\
\times(\log (|Q||\Sigma|) / 2-1)\end{array}$ & $(4 N \log (|Q|) \sqrt{|Q \|| \Sigma \mid})$ & $\begin{array}{c}N\left(K_{3}^{\log 10}+2 K_{3}^{\log 5}\right. \\
+|Q \|||| \log (|Q|))\end{array}$ \\
\hline $\mathcal{S}$ 's op. & $(2|Q||\Sigma|-\log (|Q||\Sigma|)) K_{1} N$ & $2|Q||\Sigma| \log (|Q|) N$ & $N K_{3}^{2}$ \\
\hline Comm & $\begin{array}{c}N\left(\left(K_{1} / 2\right) \log ^{2}(|Q||\Sigma|)+\right. \\
+3 \log (|Q|) \log (|Q \| \Sigma|))\end{array}$ & $\begin{array}{c}N(\log (|Q||\Sigma|)+\log (|Q|)+ \\
+4+\log (\log (|Q||\Sigma|)))\end{array}$ & \\
& & \\
\hline
\end{tabular}

efficient solutions of Lipmaa [16] and Gentry-Ramzan (GR) [17, as well as a recent lattice-based protocol of Aguilar Melchor-Gaborit (AG) [23] with very light computation overhead. We replace the original OT protocol [15] in both OFAE solutions of Sections 3 and 5 by an OT protocol based on these PIR schemes.

Before presenting our analysis, we need to point out the differences between these protocols because they are based on different setups, which will require the use of different security parameters and underlying operations. More precisely, the Lipmaa's protocol is based on the use of a length-flexible additively homomorphic encryption scheme (such as [24]), the GR protocol uses groups with special properties (in which $\Phi$-hiding assumption holds), and the AG protocol is a lattice-based PIR scheme. Thus, to achieve as precise analysis as possible, we measure the computation overhead in the number of group operations, and describe what a group operation involves in each solution.

The complexity analysis of the original OFAE approach (except the matrix multiplication in step 3 of the $k$ th state transition protocol in Section 3 ) is given in Table 2, where work is measured in group operations. The matrix multiplication cost (which is the same regardless of the OT scheme used) is given below:

\begin{tabular}{|l|c|}
\hline & Matrix Multiplication \\
\hline $\mathcal{C}$ 's group op. & $(|\Sigma|+2) K_{e} N$ \\
\hline $\mathcal{S}$ 's group op. & $N|Q| K_{e}(\log (|Q|)|\Sigma|+|\Sigma|-1)$ \\
\hline Comm & $N K_{e}(|Q|+|\Sigma|)$ \\
\hline
\end{tabular}

Similarly, Table 3 presents analysis of our modified scheme. In the tables, $K_{1}$, $K_{2}$, and $K_{3}$ are security parameters for each scheme and $K_{e}$ is the security parameter for the homomorphic encryption scheme (i.e., $K_{e}=\kappa$ ). In Lipmaa's solution, $K_{1}$ is the same as $K_{e}$, and thus is near 1024 ([25] also reports that in the Lipmaa's PIR the sender's computation could be reduced by almost $38 \%$ through optimization). In GR approach, $K_{2}$ is a parameter of a similar length, but it also depends on the configuration of the OT protocol for which it is used. 
In particular, $K_{2}=\max (\kappa, \ell, f(\log (t)))$ for $\mathrm{OT}_{1}^{t}$, where $\ell$ is the size of an element in the OT protocol and $f(\cdot)$ is a polynomial function. $K_{2}$ is not used in the tables, but it determines the cost of the group operation (multiplication modulo $K_{2}$-bit numbers). Note that in the original solution, the OT protocol is called on blocks of size $2 \kappa$, and to reduce the overhead associated with high $K_{2}$, each block can be partitioned into several blocks of smaller size (which results in executing the OT more than once).

In the AG solution, the security parameter $K_{3}$ is suggested to be set to 50 , but the group operations are performed using elements in $\mathbb{Z}_{p}$ for prime $p$ of size $3\left(\left\lceil\log \left(t K_{3}\right)\right\rceil+1\right)$ on the database of size $t$. Note that the value of $t$ in $\mathrm{OT}_{1}^{t}$ is different in the original and modified solutions $(|Q|$ and $|Q||\Sigma|$, respectively), which will affect the overhead of group operations when they depend on $t$.

From these options, the AG solution has the highest communication cost (which can be further increased to lower the computation), but it is very computation efficient unlike other protocols (also see [26] for further discussion). Thus, it is ideally suited for parties with very fast communication links. The GR approach, on the other hand, has the lowest communication cost, although the amount of computation carried on the server side as well as the client side are more pronounced. Thus, the first two methods based on Lipmaa's and GR PIR schemes should be used when the bandwidth is an issue of consideration, while the third approach gives the fastest performance with respect to the execution time assuming a fast data link between the participants.

\section{Secure Outsourcing of FSM Computation}

Secure two-party outsourcing. The idea behind this solution is that the client $\mathcal{C}$ additively splits (modulo $|\Sigma|$ ) each character of its $x$ between helper servers $P_{0}$ and $P_{1}$. Likewise, $\mathcal{S}$ splits (modulo $|Q|$ ) each element of its matrix $\Delta$ between $P_{0}$ and $P_{1}$. We refer to the $P_{i}$ 's share (for $i=0,1$ ) of string $x$ as $x^{(i)}$ and its share of the $k$ th character of $x$ as $x_{k}^{(i)}$. Similarly, we refer to the $P_{i}$ 's share of $\Delta$ as $\Delta^{(i)}$ and its share of the element of $\Delta$ at position $\left(j_{1}, j_{2}\right)$ as $\Delta^{(i)}\left(j_{1}, j_{2}\right)$. The helper servers are also given $q_{0}$, i.e., they know what row in the matrix is the start state (which gives no information about the automaton itself). Finally, $P_{0}$ and $P_{1}$ receive information about final states $F$ in a split form. We represent $F$ as a bit vector of length $|Q|$ that has $j$ th bit set to 1 iff state $j \in F$. This vector is additively split modulo 2 (i.e., XOR-split) between $P_{0}$ and $P_{1}$.

During the $k$ th state transition, $P_{0}$ acts as $\mathcal{S}$ in the previous solution and $P_{1}$ as $\mathcal{C}$, except that the share of the matrix $P_{0}$ possesses is rotated by both $P_{0}$ 's share of the next input character $x_{k}^{(0)}$ and its share of the current state $q_{k}^{(0)}$. At the end of this execution, $P_{0}$ and $P_{1}$ additively share some value $q^{\prime}$. The same steps are also performed with the roles of $P_{0}$ and $P_{1}$ reversed (using $P_{1}$ 's share of the transition matrix), which results in $P_{0}$ and $P_{1}$ additively sharing another value $q^{\prime \prime}$. Finally, $P_{0}$ and $P_{1}$ each locally add their shares of $q^{\prime}$ and $q^{\prime \prime}$, which results in state $q_{k+1}$ being split (modulo $|Q|$ ) between them. 


\section{Protocol for 1st state transition.}

1. For $i=0,1, P_{i}$ chooses value $r_{i} \stackrel{R}{\leftarrow} \mathbb{Z}_{|Q|}$, blinds each element of row $q_{0}$ by adding $r_{i}$ to it modulo $|Q|$ and rotates the row $x_{0}^{(i)}$ elements left.

2. For $i=0,1, P_{i}$ engages in $\mathrm{OT}_{1}^{|\Sigma|}$ with $P_{1-i}$, where the sender $P_{i}$ holds the modified row $q_{0}$, and receiver $P_{1-i}$ obtains the element at position $x_{0}^{(1-i)}$. Denote the element that $P_{1-i}$ receives by $s_{i}$.

3. For $i=0,1, P_{i}$ sets its share of state $q_{1}$ to $q_{1}^{(i)}=s_{1-i}-r_{i} \bmod |Q|$.

Protocol for $k$ th state transition. Prior to the protocol, $P_{0}$ and $P_{1}$ additively share the $k$ th state $q_{k}$ (modulo $\left.|Q|\right)$, the $k$ th input character $x_{k}$ (modulo $\left.|\Sigma|\right)$, and each element $\Delta(i, j)$ of $\Delta$ for $0 \leq i<|Q|$ and $0 \leq j<|\Sigma|$ (modulo $|Q|$ ). The output consists of $P_{0}$ and $P_{1}$ additively sharing the state $q_{k+1}$ modulo $|Q|$.

1. For $i=0,1, P_{i}$ chooses $r_{i} \stackrel{R}{\leftarrow} \mathbb{Z}_{|Q|}$ and adds it to each $\Delta^{(i)}\left(j_{1}, j_{2}\right) \bmod |Q|$.

2. For $i=0,1, P_{i}$ rotates the resulting matrix $\Delta^{(i)} q_{k}^{(i)}$ rows up and $x_{k}^{(i)}$ elements left, and represents it as a list of $|Q| \cdot|\Sigma|$ elements, denoted by $\Delta_{k}^{(i)}$.

3. For $i=0,1, P_{i}$ engages with $P_{1-i}$ in $\mathrm{OT}_{1}^{|Q| \cdot|\Sigma|}$ (where $P_{i}$ acts as the sender), at the end of which $P_{1-i}$ obtains the element at position $|\Sigma| \cdot q_{k}^{(1-i)}+x_{k}^{(1-i)}$ from the database $\Delta_{k}^{(i)}$ prepared by $P_{i}$. Denote the retrieved element by $s_{i}$.

4. For $i=0,1, P_{i}$ sets its share of state $q_{k+1}$ to $q_{k+1}^{(i)}=s_{1-i}-r_{i} \bmod |Q|$.

In the above $q^{\prime}=s_{0}-r_{0} \bmod |Q|$ and $q^{\prime \prime}=s_{1}-r_{1} \bmod |Q|$, and also $q_{k+1}^{(0)}=$ $s_{1}-r_{0} \bmod |Q|$ and $q_{k+1}^{(1)}=s_{0}-r_{1} \bmod |Q|$.

Protocol for announcement of result. In the beginning, $P_{0}$ and $P_{1}$ share XOR-split bit vector $F$, and at the end $\mathcal{C}$ learns the bit of $F$ at position $q_{N}$.

1. For $i=0,1, P_{i}$ generates a random bit $b_{i}$ and blinds its vector $F^{(i)}$ by XORing it with $b_{i} . P_{i}$ then rotates its $q_{N}^{(i)}$ bits left.

2. For $i=0,1, P_{i}$ engages in $O T_{1}^{|Q|}$ with $P_{1-i}$, where $P_{i}$ uses it modified vector $F^{(i)}$ as the sender and $P_{i-1}$ retrieves the bit $c_{i}$ at position $q_{N}^{(1-i)}$.

3. For $i=0,1, P_{i}$ sets it share of the result to $f^{(i)}=b_{i} \oplus c_{1-i}$.

4. $P_{0}$ and $P_{1}$ send $f^{(0)}$ and $f^{(1)}$ to $\mathcal{C}$, who XORs them and learns the result.

Secure multi-party outsourcing. To generalize the above solution to multiple parties $P_{0}, \ldots, P_{m-1}$, we first need to have $\mathcal{C}$ and $\mathcal{S}$ split their data among all parties. For a split item $a$, we use $a^{(i)}$ to denote the share party $P_{i}$ has. Since now both the input characters and the current state will be split among $m$ participants, any solution that involves data rotation by a share of the state or input character becomes more expensive. In particular, at least $m-1$ parties need to rotate the data in a predetermined order using their own shares. This means that the data to be rotated must be obfuscated from others (i.e., encrypted) when it leaves the owner and it also means that each party needs to re-randomize the data to hide the amount of rotation. With this (or any other secure) approach, the work performed by one party in a single execution of the state transition protocol is inevitably $O(|Q||\Sigma|$ ) (and is also a function of $\kappa$ ), and we wish to minimize the amount of work other parties need to perform, as well as their 
communication complexity. Therefore, we reduce the overhead of most parties to $O(\sqrt{|Q \| \Sigma|})$ by representing the transition matrix $\Delta$ as a two-dimensional array of size $\sqrt{|Q||\Sigma|} \times \sqrt{|Q||\Sigma|}$. The interaction is then similar at the highlevel to the interaction in the original protocol and proceeds as follows: one party generates a vector of encrypted bits of size $\sqrt{|Q||\Sigma|}, m-2$ parties sequentially rotate and randomize it, and the last party performs matrix multiplication to create a new vector of the same size. This vector is also passed to $m-2$ parties for rotation and re-randomization, after which the last party obtains the decryption of one element of it. This process is repeated for each share of the matrix $\Delta^{(i)}$.

Our solution requires the parties to convert shares $v^{(i)}$ of value $v$ additively split modulo $n$ to additive shares of it modulo $|Q|$. To do so, the parties will need to compute the quotient $u=\left\lfloor\sum_{i=1}^{m} v^{(i)} / n\right\rfloor$ and use it to adjust the shares. To prevent the parties from learning $u$, we additively split it over integers. Since $0 \leq u<m$, we define $B>m 2^{\kappa^{\prime}}$, where $\kappa^{\prime}$ is a security parameter. Then if we choose shares of $u$ from $[-B, B]$, the value of $u$ will be statistically hidden.

Finally, the parties now use a threshold homomorphic encryption scheme, in which the public key $p k$ is known to everyone, but the decryption key $s k$ is split among them. Here we require all $m$ parties to participate in decryption (i.e., use $(m, m)$-threshold encryption), and the threshold multi-party solution given in [10] will have the threshold set to $t$ (i.e., $(t, m)$-threshold encryption).

Before presenting the main protocols, we describe a sub-protocol, RotateAndShare, that will be utilized in all of them, but will be called on different types of data. This sub-protocol assumes that one party, $P_{i}$, has a vector, which will be encrypted, and then rotated by a certain amount, re-randomized, and blinded by every party. $P_{i}$ will be the data owner and plays a special role in the protocol. The amount of rotation is determined by some value additively split among all parties (e.g., the current state $q_{k}$ ). Blinding involves adding a random value $r_{i}$ to the encrypted contents by each party. Then when the last party chooses an element of the vector, other parties jointly decrypt that value for it. At this point, all parties jointly hold additive shares of the result modulo $n$. As the last (and optional) step, they engage in the computation to convert the additive shares modulo $n$ to additive shares modulo a different modulus $n^{\prime}$.

RotateAndShare: The input consists of value $i, 0 \leq i \leq m-1$, encryption $\mathcal{E}$ with public key $p k$, modulus $n$, and distributed secret key $s k$, final modulus $n^{\prime}$ (if no conversion is necessary, $n^{\prime}$ is set to $\left.\perp\right)$, party $P_{i}$ inputs vector $v=\left(v_{0}, \ldots, v_{\ell-1}\right)$ and its length $\ell$, and each party $P_{j}, 0 \leq j \leq m-1$ inputs amount of rotation $r t^{(j)}$. The output consists of the parties additively sharing value $o$ modulo $n^{\prime}$ (or modulo $n$ if $n^{\prime}=\perp$ ), which corresponds to one of the values from vector $v$.

1. $P_{i}$ chooses $r_{i} \stackrel{R}{\leftarrow} \mathbb{Z}_{n}$, adds it modulo $n$ to each $v_{j}$, and encrypts each result with $p k$ to obtain $e=\left(e_{0}, \ldots, e_{\ell-1}\right)$, where $e_{j}=\operatorname{Enc}_{p k}\left(v_{j}+r_{i}\right)$ for $j=$ $0, \ldots, \ell-1 . P_{i}$ circularly rotates the elements of $e r t^{(i)}$ positions left and sends the result to $P_{i+1}$.

2. $P_{i+1}$ circularly rotates the vector it received $r t^{(i+1)}$ positions left. It also chooses $r_{i+1} \stackrel{R}{\longleftarrow} \mathbb{Z}_{n}$ and multiplies each element of its resulting vector by different encryptions $\mathrm{Enc}_{p k}\left(r_{i+1}\right)$ (or by the same encryption, but then 
re-randomizes each element). This adds $r_{i+1}$ to the encrypted values. $P_{i+1}$ sends the result to $P_{i+2}$.

3. Each of $P_{i+2}, \ldots, P_{m-1}, P_{0}, \ldots, P_{i-2}$ sequentially perform the same steps at $P_{i+1}$ using their respective values of randomness $r$ and rotation amount $r t$.

4. Parties $P_{i-2}$ and $P_{i-1}$ engage in $\mathrm{OT}_{1}^{\ell}$, where the sender $P_{i-2}$ has the final encrypted vector and receiver $P_{i-1}$ uses index $r t^{(i-1)}$. This results in $P_{i-1}$ obtaining an encrypted value at position $\left(\sum_{j=0}^{m-1} r t^{(j)}\right) \bmod \ell$ in $v$ blinded with $\left(\sum_{j \in[0, m-1], j \neq i-1} r_{j}\right) \bmod n . P_{i-1}$ re-randomizes that values asks the rest of participants to decrypt it, and sets $r_{i-1}$ to the decrypted value.

5. Now, if $n^{\prime} \neq \perp$, the parties re-share the result modulo $n^{\prime}$. To do so, they compute the number of times the sum of the shares "wraps around" the modulus $n$ and use it in their computation. The parties engage in secure multi-party computation, e.g., using a standard multi-party Boolean circuit [27]. Here each party inputs its share, they jointly compute $u=\left\lfloor\left(\sum_{j=0}^{m-1} r_{j}\right) / n\right\rfloor$ (e.g., by repeated subtraction of $n$ from the sum) and the output is additively shared over the integers. That is, party $P_{j}$ for $j=0, \ldots, m-2$ receives a random $s_{j} \in[-B, B]$ and party $P_{m-1}$ receives $s_{m-1}=u-\sum_{j=0}^{m-2} s_{j}$.

6. Party $P_{j}$, for $j=0, \ldots, m-1$, sets its output $o^{(j)}$ to $\left(s_{j} \cdot n-r_{j}\right) \bmod n^{\prime}$.

We are now ready to present the main protocols of the multi-party outsourcing.

\section{Protocol for 1st state transition.}

1. For $i=0, \ldots, m-1$, execute in parallel: $P_{i}$ sets $v$ to be the $q_{0}$ th row of its $\Delta^{(i)}$ and all parties execute RotateAndShare $\left(i, \mathcal{E}, p k, s k,|Q|, v,|\Sigma|, x_{0}^{(0)}, \ldots, x_{0}^{(m-1)}\right)$. Let $o_{i}^{(j)}$ denote the output $P_{j}$ receives after such execution on $P_{i}$ 's data.

2. For $i=0, \ldots, m-1$, party $P_{i}$ sets its share of $q_{1}$ to $q_{1}^{(i)}=\sum_{j=0}^{m-1} o_{j}^{(i)} \bmod |Q|$.

Protocol for $k$ th state transition. Prior to the protocol execution, the parties additively share the $k$ th state $q_{k}$ (modulo $|Q|$ ), the $k$ th input character $x_{k}$ (modulo $|\Sigma|$ ), and each element $\Delta(i, j)$ of $\Delta$ for $0 \leq i<|Q|$ and $0 \leq j<|\Sigma|$ (modulo $|Q|$ ). At the end, they additively share state $q_{k+1}$ (modulo $|Q|$ ).

For $i=0, \ldots, m-1$, perform in parallel using the share $\Delta^{(i)}$ of $\Delta$ :

1. $P_{i}$ rotates the matrix $\Delta^{(i)} q_{k}^{(i)}$ rows up and $x_{k}^{(i)}$ elements left. We denote the resulting matrix by $\Delta_{k}^{(i)}$. $P_{i}$ represents $\Delta_{k}^{(i)}$ as a two-dimensional array of roughly square size as follows: $P_{i}$ computes the size of the first dimension of the matrix as $d_{1}=\lceil\sqrt{|Q||\Sigma|}\rceil$ and the size of the second dimension as $d_{2}=\left\lceil|Q| / d_{1}\right\rceil|\Sigma| . P_{i}$ then creates columns 0 through $|\Sigma|-1$ of the modified matrix using rows 0 through $d_{1}-1$ of $\Delta_{k}^{(i)}$, columns $|\Sigma|$ through $2|\Sigma|-1$ using rows $d_{1}$ through $2 d_{1}-1$ of $\Delta_{k}^{(i)}$, etc. In other words, the modified square matrix, denoted $\tilde{\Delta}_{k}^{(i)}$, is filled in stripes of width $|\Sigma|$ until all of $|Q|$ rows are used (note that part of the square might be incomplete due to rounding in the computation). Empty cells are then filled with dummy entries to make it a full matrix of size $d_{1} \times d_{2}$.

${ }^{1}$ In the current discussion we assume that $|\Sigma|<|Q|$, but the technique can be used when either $|\Sigma|<|Q|$ or $|Q|<|\Sigma|$. 
2. Party $P_{i+1}$ creates a vector of encrypted values $e=\left(e_{0}, \ldots, e_{d_{1}-1}\right)$ using homomorphic encryption, where the value at position $q_{k}^{(i+1)} \bmod d_{1}$ corresponds to encryption of 1 , and all other $e_{j}$ 's to encryption of 0 .

3. Party $P_{i+1}$ sends the vector to $P_{i+2}$, who performs a circular rotation of it $q_{k}^{(i+2)}$ values left and re-randomizes the encrypted values. The encrypted vector is sequentially processed by parties $P_{i+2}, \ldots, P_{m-1}, P_{0}, \ldots, P_{i-1}$ who perform the same operations as $P_{i+2}$ using their respective shares of $q_{k}$.

4. $P_{i-1}$ sends the final vector $\tilde{e}=\left(\tilde{e}_{0}, \ldots, \tilde{e}_{d_{1}-1}\right)$ to $P_{i} . P_{i}$ performs matrix multiplication using $\tilde{e}$ and $\tilde{\Delta}^{(i)}$ as follows: compute the $j$ th element of the resulting vector $v$ as $v_{j}=\prod_{\ell=0}^{d_{1}-1} \tilde{e}_{\ell}^{\tilde{\Delta}_{k}^{(i)}(\ell, j)}$. Now the vector $v$ corresponds to the element-wise encryption of the row of the matrix $\tilde{\Delta}_{k}^{(i)}$ at index $q_{k} \bmod d_{1}$.

5. All parties execute a modified algorithm RotateAndShare $\left(i, \mathcal{E}, p k, s k,|Q|, v, d_{2}\right.$, $\left.\left(x_{k}^{(0)},\left\lfloor q_{k}^{(0)} / d_{1}\right\rfloor|\Sigma|\right), \ldots,\left(x_{k}^{(m-1)},\left\lfloor q_{k}^{(m-1)} / d_{1}\right\rfloor|\Sigma|\right)\right)$ with the following changes:

(a) The vector $v$ is already in an encrypted form, so no encryption is performed in step 1 of RotateAndShare.

(b) Instead of each $P_{j}$ rotating the vector by amount $r t^{(j)}, r t^{(j)}$ now consists of two parts, $r t_{1}^{(j)}$ and $r t_{2}^{(j)}$. Starting from $j=i, P_{j}$ divides the vector $v$ into blocks of size $|\Sigma|$ and circularly rotates each block $r t_{1}^{(j)}$ positions left, and then rotates the overall resulting vector $r t_{2}^{(j)}$ positions left.

(c) Using two different values for the amount of rotation also affects the oblivious transfer in step 4 of the protocol. Now party $P_{i-1}$ selects the element at position $r t_{1}^{(i-1)}+r t_{2}^{(i-1)}|\Sigma|$.

Let $o_{j}^{(i)}$ denote the output party $P_{j}$ receives as a result of such execution. After executing these steps on all shares of the database $\Delta^{(i)}$, party $P_{j}$ sets its share of $q_{k+1}, q_{k+1}^{(j)}$, to the sum of the values it received in step 5 of the protocol executions, i.e., $q_{k+1}^{(j)}=\sum_{i=0}^{m-1} o_{i}^{(j)} \bmod |Q|$.

Protocol for announcement of result. Prior to the protocol, $P_{0}, \ldots, P_{m-1}$ additively share the state $q_{N}$ and also share vector $F$ XOR-split between them.

1. For $i=0, \ldots, m-1$, execute in parallel: the parties call RotateAndShare $\left(i, \mathcal{E}, p k, s k, \perp, F^{(i)},|Q|, q_{N}^{(0)}, \ldots, q_{N}^{(m-1)}\right)$. Let $o_{j}^{(i)}$ denote the output party $P_{j}$ receives.

2. For $i=0, \ldots, m-1, P_{i}$ computes $f^{(i)}=\sum_{j=0}^{m-1} o_{j}^{(i)} \bmod n$ and sends $f^{(i)}$ to $\mathcal{C}$.

$\mathcal{C}$ recovers the result by computing bit $b=\sum_{i=0}^{m-1} f^{(i)} \bmod n$.

The above protocol calls RotateAndShare without modulus conversion. The reason is that the client can easily recover the result by adding the shares it received modulo $n$. If the client is extremely weak, however, the above protocol can include modulus conversion from $n$ to 2 at the cost of the increased work for the helper servers. In this case, the client performs only $m-1$ bit XORs.

Also note that the protocol for announcement of the result can have a similar structure to the $k$ th state transition protocol if the vector $F$ is represented as a matrix of size $\sqrt{|Q|} \times \sqrt{|Q|}$. Then the computation and communication 
complexity of all parties will be reduced by a significant amount. But since this protocol is executed only once (as opposed to the $k$ th state transition protocol), we leave it in the simple form above.

Remark. The above technique allow us to have communication associated with processing a square two-dimensional grid to be linear in the size of its one dimension. One might ask if it might be possible to further reduce the communication by represented the matrix as a high-dimensional hypercube and still have communication to be proportional to its single dimension. Such technique was employed in private information retrieval systems to dramatically decrease communication cost to $O\left(\ell^{\epsilon}\right)$ for any $\epsilon>0$ [21] or $O\left(\log ^{2}(\ell)\right)$ [16] with stronger privacy guarantees for a database of size $\ell$. Here we note that such a solution would not work in our setting because decreasing the dimension of the matrix (represented as a hypercube of any dimension) by one requires interaction of all of the participants, and thus would involve communication close to linear in the matrix size in our case (this technique worked for PIR systems when the entire database is stored at a single location).

\section{Analysis}

We now evaluate correctness and security properties and analyze complexity.

Correctness. Correctness of the protocols follows by examination. That is, during each round of the protocol, the parties additively share the value of the next state that can be found in matrix $\Delta^{(i)}$ for each participant $P_{i}$ and add them all together to correctly share the next state. The same applies to the protocol for announcement of the result.

Security. The argument for achieving security in presence of semi-honest parties that we use here is very standard, and is based on the following components:

- The composition theorem due to Canetti 28] states that composition of secure protocols remains secure. This means that the security of the overall solution reduces to ensuring that sub-protocols or other tools used as a part of it are secure against semi-honest adversaries.

- Semantic security of homomorphic encryption ensures that no information about the underlying plaintext can be learned by observing its encryption. Threshold encryption ensures that participation of a predefined number of parties (including all parties) is necessary for decryption.

- Additive secret sharing ensures unconditional security as long as there is at least one honest party that does not collude with the rest of the participants.

Given the above, it is straightforward to build a simulator that will simulate the view of the computational parties without access to $\mathcal{C}$ 's or $\mathcal{S}$ 's data. That is, every time encryption is used, it can produce encryptions of random values that will be indistinguishable from real data due to the security property of encryption, and every time shares are used, it will also produce random shares that will be indistinguishable from the shares used in the real execution. Since only secure and composable components are used in the protocols, the overall solution is secure as well. 
Complexity. We analyze computation and communication complexity of twoparty and multi-party outsourcing protocols separately. The analysis corresponds to the $N$ executions of the $k$ th state transition protocol (as the rest of the overhead will be orders of magnitude lower).

Two-party outsourcing: The client $\mathcal{C}$ only splits its input between two servers, therefore the computation is near $N$ (no cryptography is used) and communication is $2 N \log (|\Sigma|)$. The service provider $\mathcal{S}$ splits the representation of its automaton $M$ among two servers, with the computation being near $|M|$ and communication approximately twice the size of representing $M$ (i.e., near $|Q||\Sigma| \log (|Q|))$. Each computational server incurs computation and communication overhead of both $\mathcal{C}$ and $\mathcal{S}$ in the solution with no outsourcing (as given in Table 1). That is, each server performs about $|Q||\Sigma|+N(2+|\Sigma||Q| / \kappa)$ modulo exponentiations and communicates about $2 \log (|Q|) N|\Sigma||Q|$ bits.

Multi-party outsourcing: The work and communication of $\mathcal{C}$ and $\mathcal{S}$ remain similar to the two-party case, except that splitting of their data and communication needs to be done for $m$ servers instead of two. This means that work becomes proportional to $m$ (with no cryptographic operations, as before), which for $\mathcal{C}$ means $m N$ and for $\mathcal{S}$ is $m|M|$, and their communication is $m N \log (|\Sigma|)$ and near $m|Q||\Sigma| \log (|Q|)$, respectively. The computation and communication requirements for the computational servers also now increase by a factor of $m$ and are as follows. The main computation overhead comes from (i) $2 \sqrt{|Q||\Sigma|}(m-1) \bmod -$ ular exponentiations in each round due to re-randomization; (ii) $|Q \| \Sigma| \log (|Q|)$ modular multiplications in each round for matrix multiplication; (iii) $\kappa \mathrm{OT}_{1}^{2} \mathrm{ex}-$

ecutions for the Boolean circuit and one $\mathrm{OT}_{1}^{\sqrt{|Q||\Sigma|}}$ in each round. We assume that the OT protocol with low amortized cost (one mod exp per transfer) is used. The communication complexity is dominated by the transmission of encrypted vectors and the OT protocol and is near $4 \kappa(m-1) N \sqrt{|Q||\Sigma|}$.

Acknowledgments. Portions of this work were sponsored by grant AFOSRFA9550-09-1-0223. The first author would like to thank Scott Emrich for useful discussions regarding DNA processing technology.

\section{References}

1. Atallah, M., Kerschbaum, F., Du, W.: Secure and private sequence comparisons. In: WPES, pp. 39-44 (2003)

2. Jha, S., Kruger, L., Shmatikov, V.: Towards practical privacy for genomic computation. In: IEEE Symposium on Security and Privacy, pp. 216-230 (2008)

3. Troncoso-Pastoriza, J., Katzenbeisser, S., Celik, M.: Privacy preserving error resilient DNA searching through oblivious automata. In: ACM CCS, pp. 519-528 (2007)

4. Frikken, K.: Practical private DNA string searching and matching through efficient oblivious automata evaluation. In: DBSec, pp. 81-94 (2009)

5. Bruekers, F., Katzenbeisser, S., Kursawe, K., Tuyls, P.: Privacy-preserving matching of DNA profiles. ePrint Cryptology Archive Report 2008/203 (2008)

6. Wang, R., Wang, X., Li, Z., Tang, H., Reiter, M., Dong, Z.: Privacy-preserving genomic computation through program specialization. In: ACM CCS, pp. 338-347 (2009) 
7. Atallah, M., Li, J.: Secure outsourcing of sequence comparisons. In: PET, pp. 63-78 (2004)

8. Atallah, M., Li, J.: Secure outsourcing of sequence comparisons. International Journal of Information Security 4(4), 277-287 (2005)

9. Genetic Testing for Health, Disease \& Ancestry; DNA Test - 23andMe, http://www.23andme.com

10. Blanton, M., Aliasgari, M.: Secure outsourcing of DNA searching via finite automata. Technical Report 2010-03, University of Notre Dame (2010)

11. Szajda, D., Pohl, M., Owen, J., Lawson, B.: Toward a practical data privacy scheme for a distributed implementation of the Smith-Waterman genome sequence comparison algorithm. In: NDSS (2006)

12. Kantarcioglu, M., Jiang, W., Liu, Y., Malin, B.: A cryptographic approach to securely share and query genomic sequences. IEEE Transactions on Information Technology in Biomedicine 12(5), 606-617 (2008)

13. Franklin, M., Gondree, M., Mohassel, P.: Communication-efficient private protocols for longest common subsequence. In: RSA, pp. 265-278 (2009)

14. Gondree, M., Mohassel, P.: Longest common subsequence as private search. In: WPES, pp. 81-90 (2009)

15. Naor, M., Pinkas, B.: Efficient oblivious transfer protocols. In: SODA, pp. 448-457 (2001)

16. Lipmaa, H.: An oblivious transfer protocol with log-squared communication. In: Zhou, J., López, J., Deng, R.H., Bao, F. (eds.) ISC 2005. LNCS, vol. 3650, pp. 314-328. Springer, Heidelberg (2005)

17. Gentry, C., Ramzan, Z.: Single-database private information retrieval with constant communication rate. In: ICALP, pp. 803-815 (2005)

18. Naor, M., Pinkas, B.: Oblivious transfer and polynomial evaluation. In: STOC (1999)

19. Crescenzo, G., Malkin, T., Ostrovsky, R.: Single database private information retrieval implies oblivious transfer. In: Preneel, B. (ed.) EUROCRYPT 2000. LNCS, vol. 1807, pp. 122-138. Springer, Heidelberg (2000)

20. Cachin, C., Micali, S., Stadler, M.: Computationally private information retrieval with polylogarithmic communication. In: Stern, J. (ed.) EUROCRYPT 1999. LNCS, vol. 1592, pp. 402-414. Springer, Heidelberg (1999)

21. Kushilevitz, E., Ostrovsky, R.: Replication is not needed: Single database, computationally-private information retrieval. In: IEEE FOCS, pp. 364-373 (1997)

22. Melchor, C.A., Deswarte, Y.: Single-database private information retrieval schemes: Overview, performance study, and usage with statistical databases. In: Privacy in Statistical Databases, pp. 257-265 (2006)

23. Aguilar-Melchor, C., Gaborit, P.: A lattice-based computationally-efficient private information retrieval protocol. In: WEWORC (2007)

24. Damgard, I., Jurik, M.: A length-flexible threshold cryptosystem with applications. In: Australasian Conference on Information Security and Privacy (2007)

25. Bae, H.: Design and analysis for log-squared and log private information retrieval (2008)

26. Melchor, C., Crespin, B., Gaborit, P., Jolivet, V.: High-speed private information retrieval computation on GPU. In: IEEE SECURWARE (2008)

27. Goldreich, O., Micali, S., Wigderson, A.: How to play any mental game. In: STOC, pp. 218-229 (1987)

28. Canetti, R.: Security and composition of multiparty cryptographic protocols. Journal of Cryptology 13(1), 143-202 (2000) 